NOW AND THEN

\title{
A method for estimating macromolecular reflection by human synovium, using measurements of intra-articular half lives
}

\author{
J R Levick
}

Department of Physiology, St George's Hospital Medical School, Cranmer Terrace, Tooting, London SW17 0RE

Correspondence to: Professor Levick. 8 April 1998
Accepted for publication

\begin{abstract}
Recent studies show that very large macromolecules in synovial fluid, such as hyaluronan and large proteoglycans, are partially reflected by the synovial lining during fluid drainage, and thus selectively retained within the cavity. Size selective molecular reflection is a fundamental property of membranes, and a method for quantifying the reflective behaviour of human synovium could be of value in several pathophysiological areas. The method proposed here is based on the intra-articular half lives of the macromolecule of interest and of a smaller, easily cleared protein such as albumin. The key relation is derived from the law of conservation of mass, using simple algebra. It is found that, when the intra-articular half lives of albumin and a macromolecular test solute are determined simultaneously, the reflected fraction of the test solute is given
\end{abstract}

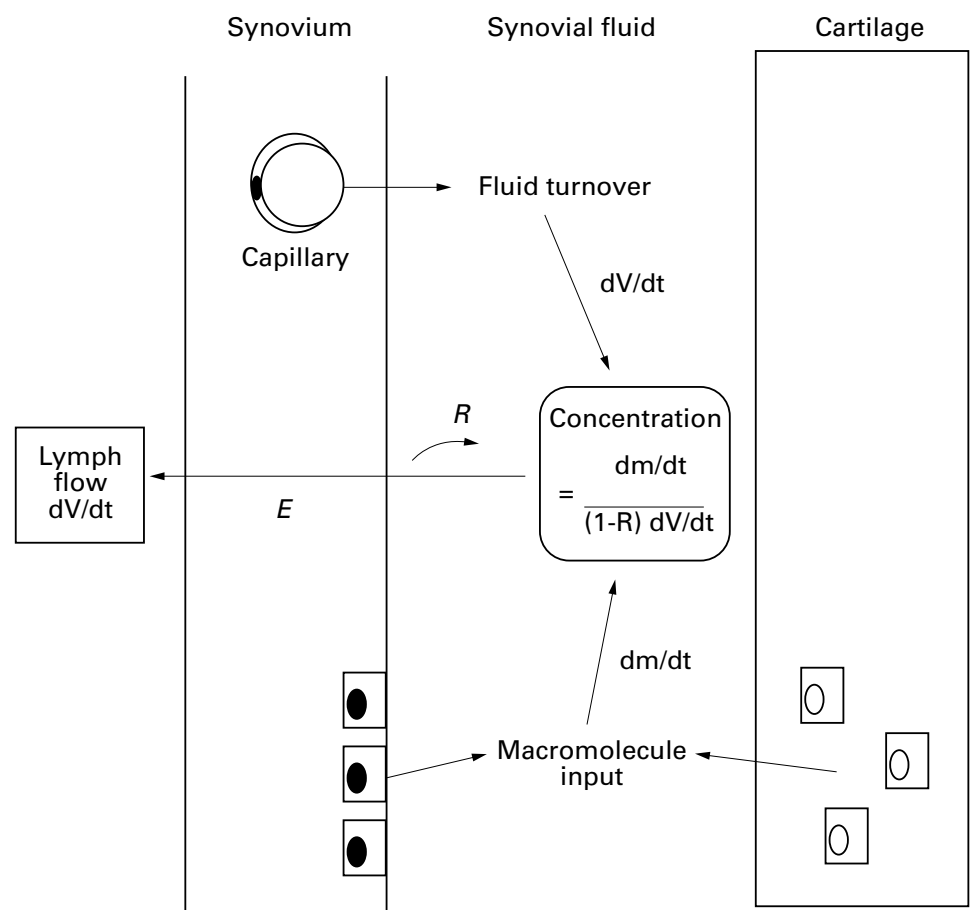

Figure 1 Schematic diagram to illustrate relation between intra-articular concentration of a macromolecule, fluid turnover rate $(d V / d t)$ and partial molecular reflection at the synovial surface. The term dm/dt is the rate of entry of the macromolecule into the synovial cavity, $R$ is the fraction of the macromolecules in the escaping fluid that is reflected and $E$ is the escaping fraction. by the complement of the half life ratio. Examples are given. Intra-articular half lives can thus be used to consider such questions as whether immune complexes are significantly reflected by the synovial surface, how the reflective property changes in arthritides or with treatment, and how significantly reflection might influence the intra-articular concentration of large "markers" of joint disease activity. (Ann Rheum Dis 1998;57:339-344)

Background; animal studies of synovial reflection of hyaluronan (fig 1)

When a solution flows under pressure across a porous membrane, the pores may reflect some or all of the solute molecules back into the filtrand (upstream bulk phase), if the pore width is smaller or comparable in size to solute width, leaving the filtrate depleted of the solute. ${ }^{12}$ This is called "molecular sieving" or "ultrafiltration". Size dependent molecular sieving is a characteristic and important property of porous membranes, but little is known about it in the case of synovium. Indeed, synovium has even been considered a non-reflective membrane, because there are $\mu \mathrm{m}$ wide interstitial spaces between the lining cells, and because synovial fluid/serum ratios for plasma proteins seemed compatible with passive interstitial diffusion. ${ }^{3}$

It is clear from older work with particulate injections that there is an upper limit (40-250 $\mathrm{nm}$ radius) to the size of particles that can escape freely from the joint cavity. ${ }^{4}$ Recent work on rabbit joints showed this to be also the case for very large solutes, because it was found that the intra-articular lubricant hyaluronan (radius of gyration $100-200 \mathrm{~nm}$ ) is partially reflected by the synovial lining. Depending on the hyaluronan concentration, between $48 \%$ and $95 \%$ of the hyaluronan molecules in synovial fluid filtering out of the joint cavity are reflected back and retained within the cavity. ${ }^{56}$ Lubricant is thereby conserved while water and albumin escape through the lining. In this way the concentration of hyaluronan is maintained at the high levels that characterise healthy synovial fluid.

Potential value of a method applicable to humans

There are several circumstances where information on the reflection of large macromolecules by human synovium could be of pathophysiological value. For example, (1) a comparison of 
macromolecular reflection by rheumatoid compared with healthy human synovium would increase our understanding of the pathophysiology of synovial effusions, would quantify the changes in the state of the synovial lining brought about by the disease, and also allow quantification of synovial responses to drug treatment. (2) In interpreting the concentration of "markers of joint disease activity" in synovial fluid, it is generally assumed, without explicit declaration (and despite the older literature on particle retention), that the marker experiences zero reflection by the joint lining during clearance into the subsynovial lymph vessels. This is probably true for relatively small markers like keratan sulphate. Some "markers", however, are large macromolecules, for example, proteoglycans. If, like hyaluronan, they are partially reflected by the lining to some degree (fig 1), marker concentration may be influenced by the health of the synovial lining as well as by that of cartilage. $^{78}$ This issue needs resolving. (3) A method for determining synovial reflection in humans will allow quantification of the retention of large immune complexes within joints. The ability to do so may prove important in view of the proposal that retention of giant hyaluronan complement-IgG aggregates in rheumatoid synovial fluid is a factor in disease chronicity. ${ }^{9}$

There are many pathophysiological reasons, therefore, for developing a way of quantifying the synovial reflection of large macromolecules in humans. The experimental method used by Scott $e t a l^{6}$ in anaesthetised animals may not be practical in humans. Insights gained from the animal work, however, led to the realisation that reflection can, in principle, be measured in humans by comparing the intra-articular half lives of the test macromolecule and a reference substance, radiolabelled albumin. The reasoning is explained here, and examples of its application are given from animal work.

\section{Theory; determination of reflected fraction by comparing intra-articular half lives}

The volume of fluid in a joint cavity is replaced many times over 24 hours - that is, fresh saline and plasma proteins enter from the synovial microcirculation and the resident synovial fluid (minus much of hyaluronan) filters out into the subynovial lymphatics. ${ }^{6}{ }^{10}$ Let the fraction of large macromolecules (for example, large proteoglycans, immune complexes) that is sieved out of the fluid leaving the joint cavity be R; that is to say, fraction $\mathrm{R}$ is reflected by the lining and thus retained in the cavity. This fraction can be determined by measuring the intra-articular half life of the "test" macromolecule and comparing it with the intra-articular half life of an essentially unreflected "reference" solute, such as albumin. The half life of a solute can be determined in practice by intra-articular injection of radiolabelled solute followed by external counting. The reasoning behind this "half life ratio" method is as follows.

TURNOVER TIMES AND HALF LIVES

The time required to replace the entire mass $(m)$ of a macromolecule in the synovial fluid is $m$ divided by the rate of entry of that solute into the fluid, $d m / d t$ (the "little bit" of solute mass $\mathrm{dm}$ that passes in little bit of time, dt). This is called the turnover (or residence) time, $\left(t_{T}\right)$.

$$
\text { Turnover time, } t_{T}=m /(d m / d t)
$$

For example, given $182 \mu \mathrm{g}$ of hyaluronan in the synovial fluid of a rabbit knee joint, and a hyaluronan secretion rate of $4.8 \mu \mathrm{g} / \mathrm{h}$, the turnover time is 37.9 hours. ${ }^{6}$ The fraction of the entire mass that is replaced in unit time is $1 /$ $t_{T}$. This fractional "clearance" rate is called the removal rate constant, $k$. The half life of the solute in the synovial fluid, $t_{1 / 2}$, is $0.693 / k$, or $0.693 . t_{T}$, because -0.693 is the natural logarithm of $1 / 2$. Thus, from equation [1]

$$
\text { Half-life, } t_{1 / 2}=0.693 \mathrm{~m} /(\mathrm{dm} / \mathrm{dt})
$$

This is an algebraic statement of the standard definition of half life, namely the time taken to lose half the material.

\section{CONCENTRATION OF MACROMOLECULE IN THE}

STEADY STATE. WHAT DETERMINES IT?

To work out the relation between intraarticular half life (equation 2) and reflected fraction, we must consider what determines the concentration of a macromolecule in synovial fluid in the steady state. This is also an important relation in its own right, for those interested in immune complex concentration, lubricant concentration, markers of joint disease activity, etc. The analysis applies to periods when the concentration is stable (the steady state), or equivalently to the average concentration over a timescale of hours, where minute to minute fluctuations caused by joint movement, blood pressure, and intra-articular pressure variations are averaged out.

In a steady state, the average rate of entry of macromolecules into the joint cavity (for example, hyaluronan secretion rate; release of proteoglycan from cartilage; $d m / d t$ ) must be matched by their rate of removal. Macromolecular removal from joints is chiefly by drainage of the fluid across the synovial lining into the subsynovial lymphatic system. ${ }^{11-17}$ (It is assumed, in absence of evidence to the contrary, that macrophagic removal is slow compared with lymphatic clearance; but see discussion of this in caveat 2, below.) Let the mean fluid drainage rate (flow) over the period of interest be $d V / d t$ (the "little bit" of volume, $\mathrm{dV}$, that passes in a little bit of time, $\mathrm{dt}$ ) - that is to say, this is the average volume turnover rate. Also, let $R$ be the average fraction of test macromolecules in the draining fluid that is reflected by the synovial lining and thus retained in the joint cavity, over the same period. The reflected fraction $R$ can be any value less than 1, down to 0 . The fraction $E$ that escapes into the lymph, by the combined processes of fluid drainage (bulk flow or "convective" transport) and diffusion, is $1-R$. The average escape rate for test solute is, therefore, $C \times E \times d V / d t$, where $C$ is concentration in synovial fluid (mass per unit volume of solution; previously erroneously defined as mass/available space ${ }^{8}$ ). 
By the law of conservation of mass, macromolecule entry rate $=$ removal rate, in the steady state. Substituting the expressions from the previous paragraph for entry rate and removal rate into the above law, we find that intraarticular concentration $C$ is governed by the mass input rate, the escaping fraction and the volume turnover rate;

Concentration of macromolecule in a turningover fluid, $C=(d m / d t) /(E d V / d t)$

Expression [3a] is a fundamental one, as it governs the concentration of immune complex, lubricant, "marker", etc, in synovial fluid. $\mathrm{Re}$-arrangement gives the escaping fraction:

Escaping fraction, $E=(d m / d t) /(C d V / d t)$ [3b]

USE OF A REFERENCE SOLUTE (FLOW MARKER) TO ESTIMATE VOLUME TURNOVER RATE, $d V / d t$

A second solute, the reference solute, is needed to provide information about the time averaged trans-synovial flow, $d V / d t$. Simkin and others have shown that the magnitude of $d V / d t$ can be determined, in human joints, by the rate of removal from the joint cavity of a flow marker such as labelled albumin. ${ }^{11-13}$ Albumin washes out through the lining with the draining fluid, ${ }^{12-16}$ and reflection of albumin by synovium is for practical purposes negligible $(E$ $\approx 1^{17}$ ), so albumin. is a suitable flow marker. Applying expression [3a] to the case of albumin, where $E \approx 1$, and rearranging, we find that:

Volume turnover rate, $d V / d t=(d m / d t)_{a l b} / C_{a l b}[4]$

where $C_{a l b}$ is albumin concentration in synovial fluid and $(d m / d t)_{a l b}$ is the corresponding intra-articular mass turnover rate for albumin.

COMBINING THE ABOVE RELATIONS TO GET THE

REFLECTED FRACTION, $R$

If the albumin mass in the synovial fluid is $m_{a l b}$, we see from [2] that albumin turnover rate $(d m / d t)_{a l b}$ is $0.693 m_{a l b} / t / s, a l b$. Substituting this into [4], and noting also that intra-articular albumin mass $m_{a l b}$ is albumin concentration $C_{a l b}$ $\times$ synovial fluid volume $V_{s f}$, we find that the $C_{a l b}$ terms cancel out. The volume turnover rate, in terms of albumin half life, is thus:

$$
d V / d t=0.693 V_{s f} / t_{1 / 2, a l b}
$$

Denoting the amount of macromolecules of interest (the "test" molecules) in the synovial fluid as $m_{\text {test }}$, equation [2] tells us that the turnover rate for the test macromolecule, $(\mathrm{dm} /$ $d t)_{\text {test }}$, equals $0.693 m_{\text {tess }} / t_{1 / 2, \text { test. }}$. As the intraarticular solute mass $m_{\text {test }}$ is concentration $C_{\text {test }} \times$ synovial fluid volume $V_{s f}$, expression [2] tells us that test macromolecule's turnover rate is related to its half life thus:

$$
(d m / d t)_{\text {test }}=0.693 C_{\text {test }} V_{s f} / t^{1 / 2, \text { test }}
$$

Expressions [5] and [6] contain the intraarticular half lives of the test and reference solutes, so we can now discover what their ratio signifies. The two expressions are substituted into [3b] to find the escaping fraction of test solute, $E$. If the half lives are measured under identical conditions, the synovial fluid volumes
$V_{s f}$ in equations [5] and [6] are identical. They then cancel out on substitution into [3b], leading to a very simple relation between escaping fraction and half lives:

$$
\begin{aligned}
& \text { Escaping fraction of test solute, } \\
& E=\left\{t^{1 / 2, \text { alb }} / t^{t / 2, \text { test }}\right\} \\
& \text { and Reflected fraction of test solute, } \\
& R=1-\left\{t_{1 / 2, \text { alb }} / t_{1 / 2, \text { test }}\right\}
\end{aligned}
$$

Thus a very simple and intuitively correct relation emerges. The escaping fraction of the test macromolecule is equal to the ratio of albumin half life to test solute half life. In other words, the longer the test solute's half life, relative to albumin, the smaller the escaping fraction and the greater the reflected fraction.

\section{Caveats}

The following conditions must be met for the above reasoning, and therefore the method, to be valid.

(1) The test solute must be cleared by drainage into the lymphatic system, not by direct diffusion into the synovial capillary blood stream. The method therefore applies to macromolecules that are known to clear from the interstitial compartment via the lymphatic system, and not by diffusion across the capillary wall, which is relatively impermeable to macromolecules. ${ }^{12}$ The method does not apply to small molecules like cytokines, lactate, neuropeptides, autacoids, etc, which diffuse easily across the capillary wall and are therefore cleared chiefly by the blood stream. These have an escape factor $E$ of $>1$ and hence an apparent negative reflected fraction (see below).

(2) The macromolecule of interest should not, ideally, be degraded within the joint to a significant degree, for example, by macrophages. While there is clear evidence that synovial macrophages take up particles and some macromolecules, the rate of macrophagic uptake seems never to have been compared with the rate of lymphatic clearance. In most tissues, however, lymphatic clearance is accepted as the dominant macromolecular removal process (based on the effect of reduced lymph flow), and the same is assumed here for joints. The method still has value if synovial macrophages turn out to account for a significant fraction of (say) a marker's removal, in that the true reflected fraction will be bigger than calculated $R$; the method then provides a useful minimal estimate of $R$. If an $R$ value of $<0$ (negative value, that is, $E>1$ ) for a macromolecule were ever found, this could indicate that macrophagic removal was the dominant clearance process.

(3) The measurements of test and reference solute half lives should be made at identical synovial fluid volumes if expression $[7 \mathrm{a} / \mathrm{b}]$ is to be used, because only then do the volume terms in [5] and [6] cancel out. Ideally, therefore, measurements should be made in the same joint at the same time. A further advantage of doing so is that the estimate of average fluid turnover rate, namely albumin's intra-articular half life, is made at the same time as the test macromolecule measurement. 
Table 1 Reflected and escaping fractions of large macromolecules across synovium, calculated from intra-articular half lives using equation $7 a / b$

\begin{tabular}{|c|c|c|c|c|c|}
\hline \multirow[b]{2}{*}{ Source } & \multirow[b]{2}{*}{ Test macromolecule } & \multicolumn{2}{|c|}{$t^{1 / 2}$ Intra-articular half life $(h)$} & \multirow[b]{2}{*}{$\begin{array}{l}R \\
\text { Reflected fraction }\end{array}$} & \multirow[b]{2}{*}{$\begin{array}{l}E \\
\text { Escaping fraction }\end{array}$} \\
\hline & & $\begin{array}{l}\text { Test } \\
\text { macromolecule }\end{array}$ & $\begin{array}{l}\text { Flow marker } \\
\text { (albumin) }\end{array}$ & & \\
\hline Page-Thomas et al ${ }^{18}$ & Large cartilage proteoglycan & $12.5(0.7)$ & $3.9(0.8) \mathrm{h}$ & 0.69 & 0.31 \\
\hline Coleman $e t a \bar{P}$ & Native synovial hyaluronan & $26.3 \mathrm{~h}$ & $1.23 \mathrm{~h}$ & 0.95 & 0.05 \\
\hline
\end{tabular}

Data shown as mean (SEM).

Failing this, the synovial fluid volumes in separate joints, namely $V_{s f}$ test and $V_{s f}$ alb , must be measured individually, for example by an indicator dilution method. The solution from expressions [5] and [6] is then:

$$
\begin{aligned}
& \text { Escaping fraction } E= \\
& \left(t_{1 / 2, \text { alb }} / t_{1 / 2 \text { test }}\right) \cdot\left(V_{\text {sf test }} / V_{\text {sf all }}\right)
\end{aligned}
$$

\section{Application of the half life ratio method; examples (table 1)}

APPLICATION 1 ; PROTEOGLYCAN DATA OF

PAGE-THOMAS AND COLLEAGUES ${ }^{18}$

Page-Thomas and colleagues ${ }^{18}$ studied the rate of removal of radiolabelled proteoglycan of molecular weight $2.5 \times 10^{6} \mathrm{Da}$ and radiolabelled albumin (Mw 67 000) from normal rabbit joints. They found that the intra-articular half life of the proteoglycan averaged 12.5 hours and that of albumin averaged 3.9 hours (table 1). As the rabbit joints were normal, and standard injection volumes $(0.4 \mathrm{ml})$ were used, it is reasonable to assume that caveat 3 was not contravened. Applying equation [7b] to these data:

$$
R_{\text {proteoglycan }}=1-(3.9 / 12.5)
$$

The result is a rejected fraction $R$ of 0.69 . Synovial reflection of a large proteoglycan is thus substantial. This will significantly influence the intra-articular concentration of this marker of

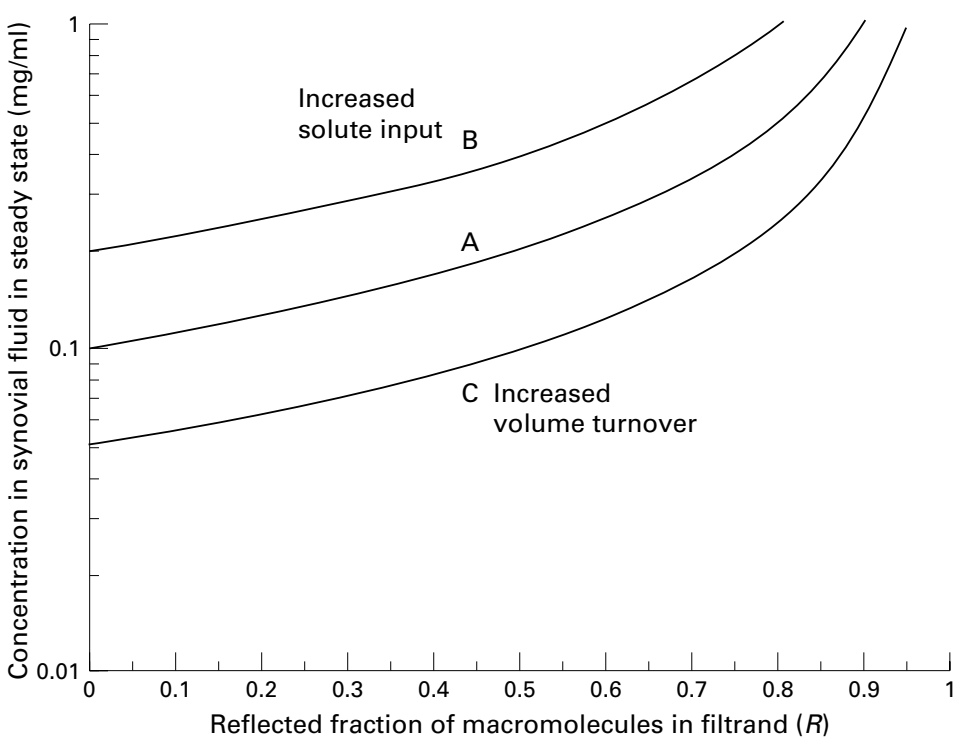

Figure 2 Effect of reflected fraction $R$ upon the concentration of a macromolecule in synovial fluid. Based on law of conservation of mass, equation [3a]. Curve A shows concentrations for a macromolecule that enters the joint at a rate $(d \mathrm{~m} / \mathrm{dt})$ of $0.1 \mathrm{mg} / \mathrm{h}$, with an accompanying volume turnover rate $(\mathrm{dV} / \mathrm{dt})$ of $1 \mathrm{ml} / \mathrm{h}$. Its concentration in the steady state is $0.1 \mathrm{mg} / \mathrm{ml}$ if the reflected fraction is zero, but $1 \mathrm{mg} / \mathrm{ml}$ if the reflected fraction is 0.9 . Curve B shows the effect of doubling the macromolecular input rate, or of halving the fluid turnover rate. Curve $C$ shows the effect of doubling the fluid turnover rate, as commonly happens in arthritides. ${ }^{15}$ cartilage disease activity, as stated in equation [3a]. Figure 2 shows the effect of rejected fraction on concentration in synovial fluid.

The authors also measured the half life of sodium pertechnate, namely 0.82 hours. This small molecule illustrates the need for caveat 1. Pertechnate contravenes caveat 1 because, unlike a macomolecule, it can diffuse directly into the bloodstream and is cleared quicker by this route than by lymphatic drainage. If the calculation of "escaping fraction" were carried out (equation [7a] ), a "nonsensical" $E$ greater than 1 would be obtained $(E=4.8, R=-3.8)$. Such results signal to the investigator that most of the solute removal is not by fluid drainage into lymph but by diffusion into synovial capillaries (for small solutes) or perhaps by macrophagic degradation (for macromolecules).

\section{APPLICATION 2; HYALURONAN DATA OF COLEMAN} AND COLLEAGUES ${ }^{5}$

The input rates $(d m / d t)$ and endogenous masses $(m)$ of hyaluronan and albumin in the synovial fluid have been measured directly in normal rabbit knees. ${ }^{5}$ From equation [2], the intra-articular half lives were found to be 26.3 hours for native hyaluronan (Mw about $3 \times 10^{6}$ Da) and 1.23 hours for albumin (table 1). No volume expansion of the cavity was involved, so caveat 3 was not contravened. Thus from equation [7b]:

$$
R_{\text {hyaluronan }}=1-(1.23 / 26.3)
$$

The result is a rejected fraction of 0.95 . Hyaluronan is, therefore, substantially retained within the joint cavity, raising its concentration there (fig 2) and enabling it to fulfil its important physiological roles. The high reflected fraction is thought to be a consequence of the enormous size of the hydrated molecular domain, the radius of which is about $200 \mathrm{~nm}$ (compared with $3.55 \mathrm{~nm}$ for albumin), and also of the entangled state of adjacent chains at physiological concentration.

A substantial volume expansion was inherent in the method of Page-Thomas et al, ${ }^{18}$ namely from the endogenous volume of $50 \mu \mathrm{l}$ to an injected volume of $400 \mu \mathrm{l}$. This probably contributed to their slow albumin removal rate constant $(k)$, because the removal rate constant depends on volume as well as removal rate. ${ }^{71112}$

\section{Discussion}

The examples in table 1 illustrate the potential of the method. They also indicate that, at least for some very large macromolecules found in synovial fluid, there is substantial reflection at the synovial surface. This is because of the limiting size of the porosities in the interstitial 


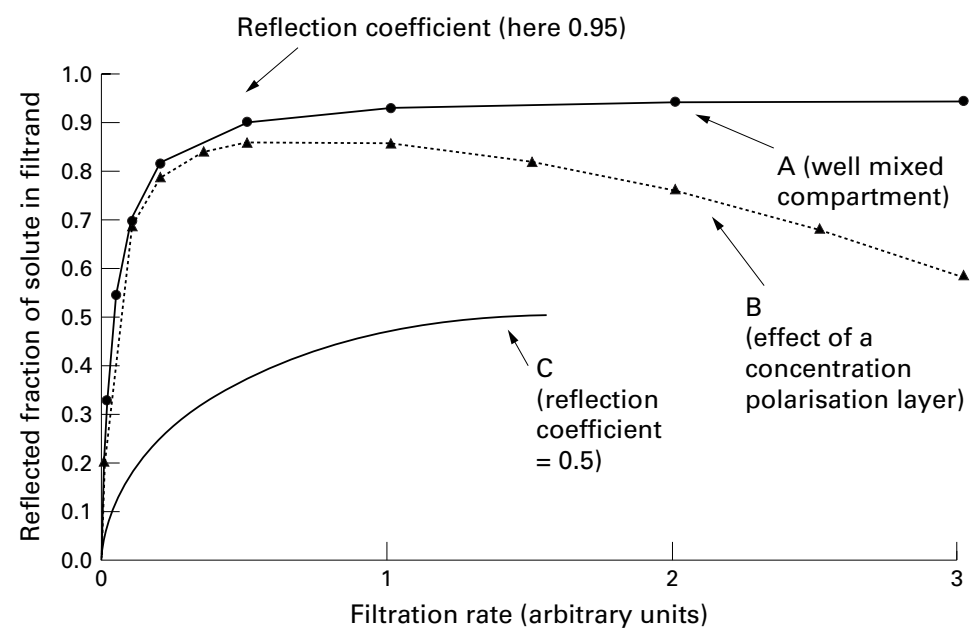

Figure 3 Relations between reflected fraction and filtration rate for a simple homoporous membrane. The reflected fraction of the solute approaches a limit, called the reflection coefficient, as filtration rate increases. Curve A. Perfectly stirred fluid upstream of membrane, and a membrane reflection coefficient of 0.95. Based on Patlak et al. ${ }^{20}$ Curve $B$. The effect of a concentration polarisation layer developing near a partially reflective membrane in a poorly stirred cell. This causes the relation to deviate at high filtration rates. Curve C. As for curve A but for a membrane of reflection coefficient 0.5.

matrix of the lining. ${ }^{19}$ Significant levels of reflection of synovial fluid macromolecules by the joint lining will substantially increase their concentration in synovial fluid. This is illustrated in figure 2, where the concentration of macromolecules in synovial fluid is plotted for various reflected fractions, $R$.

The reflected fraction attains the same value as the reflection coefficient only at high flows. The reflected fraction varies with flow across the membrane, according to both experiment and theory. ${ }^{121920}$ Trans-synovial flow is measurable by Simkin's albumin clearance method in human joints. ${ }^{11-13}$ Figure 3 shows the general form of the relation between reflected fraction and transmembrane flow. The reflected fraction is zero at zero filtration rate, because diffusion produces equilibration across the membrane when there is no flow to wash away even a slowly permeating solute from the far side of the membrane; and the reflected fraction increases non-linearly with filtration rate, up to a sieving limit. ${ }^{120}$ The reflection coefficient is the maximum reflected fraction (dimensionless) and is an index of the size of the pores relative to the size of the solute. The reflection coefficient is one of three coefficients needed to characterise fully any porous membrane, the other two being "permeability" (describing ease of permeation by diffusion in the absence of flow) and "hydraulic conductance" (describing purely hydraulic flow, and sometimes referred to as hydraulic permeability). ${ }^{1}$ Unlike permeability or hydraulic conductance, the reflection coefficient does not depend on pore numbers, only size.

The above derivation $[7 \mathrm{a} / \mathrm{b}]$, was based on the endogenous half lives of albumin and test macromolecule, such as those measured in the rabbit knee. ${ }^{5}$ If half lives are determined by injecting tracer quantities of exogenous, labelled albumin and test molecule, followed by external counting, there will initially be a labelled albumin concentration gradient between joint cavity and subynovium. Labelled albumin clearance from joint cavity to subsynovium will initially be by diffusion, therefore, as well as convection - that is, labelled albumin will not act as a pure flow marker in the first minutes after injection $(E>1)$. In a relatively short time $(\leqslant 30$ minutes), however, the concentrations of labelled albumin in cavity and subsynovium equilibrate $^{21}$ and subsequent albumin transport out of the cavity is convective-that is, disappearance of labelled albumin counts from the scanned joint segment is a flow marker at later times.

\section{Conclusion}

The key relation derived here, equation [7], is a simple algebraic derivation from the law of conservation of mass. It shows that when the half lives of albumin and another macromolecular test solute are determined simultaneously, or separately at known synovial fluid volumes, the reflected fraction of the test solute is given by the complement of the half life ratio. A study of half lives in joints can therefore be used, in principle, to consider pathophysiological issues such as whether a test macromolecule is significantly reflected by the human synovial surface, how that reflection changes in various disease states, how it responds to various treatments, and how significantly it might influence the intra-articular concentration of a "marker of joint disease activity".

ADDENDUM - Note added in proof. It was recently estimated that about $25 \%$ of a radiolabelled form of hyaluronan is taken up by cells arond the rabbit knee, while the remainder clears slowly via the lymphatic system. (Fraser JRE, Laurent UBG. The turnover of hyaluronan. In: Read RK, Rubin $\mathrm{K}$, eds. Connective tissue biology. London: Portland Press, 1998:49-68).

1 Levick JR. Transport processes: diffusion, convection, reflection, In: Levick JR. An introduction to cardiovascular physiology. 2nd ed. Oxford: Butterworth-Heineman, 1995: physiology. 2nd ed. Ox

2 Curry FE. Mechanics and thermodynamics of transcapillary exchange. In: Renkin EM, Michel CC, eds. Handbook
of physiology. Section 2. The cardiovascular system. Vol IV. Bethesda: American Physiology Society, 1984:309-74.

3 Simkin PA. Synovial physiology. In: McCarty DJ, ed. Arthritis and allied conditions. Philadelphia: Lea and Febiger, 1985: 196-209.

4 Levick JR. Blood flow and mass transport in synovial joints. In: Renkin EM, Michel CC. Handbook of physiology. Section . The cardiovascular system. Vol IV. The microcirculation. Bethesda: American Physiology Society, 1984:917-47.

5 Coleman PJ, Scott D, Ray J, Mason RM, Levick JR. Hyaluronan secretion into the synovial cavity of rabbit knees and comparison with albumin turnover. J Physiol 1997;503:645-56.

6 Scott D, Coleman PJ, Mason RM, Levick JR. Direct evidence for the partial reflection of hyaluronan molecules by the lining of joints during trans-synovial flow. J Physiol 1998; $508: 619-28$

7 Levick JR. The 'clearance' of macromolecular substances such as cartilage markers from synovial fluid and serum. In: Maroudas A, Kuettner K, eds. Methods in cartilage research. London: Academic Press, 1990:352-7.

8 Levick JR. Synovial fluid; determinants of volume and material concentration. In: Kuettner KE, Hascall VC, Schleyerbach R, eds. Articular cartilage and osteoarthritis. New York: Raven Press, 1992:529-41.

9 Prehm P. Synovial hyaluronate in rheumatoid arthritis binds $\mathrm{C} 1 \mathrm{q}$ and is covalently bound to antibodies; a model for chronicity. Ann Rheum Dis 1995;54:408-12.

10 Levick JR. Fluid movement across synovium in healthy joints: role of synovial fluid macromolecules. Ann Rheum Dis $1995 ; 54: 417-23$.

11 Wallis WJ, Simkin PA, Nelp WB, Foster DM. Intra-articular volume and clearance in human synovial effusion. Arthritis

12 Simkin PA, Benedict RS. Iodide and albumin kinetics in normal canine wrists and knees. Arthritis Rheum 1990;33: 73-9. 
13 Myers SL, O'Connor BL, Brandt, KD. Accelerated clearance of albumin from the osteoarthritic knee: implications for interpretation of concentration of "cartilage markers" in synovial fluid. J Rheumatol 1996;23:1744-8.
Bauer W, Short CL, Bennett GA. The manner of removal of

14 Bauer W, Short CL, Bennett GA. The manner of removal of proteins from normal joints. J Exp Med 1933;57:419-32.

15 Wallis WJ, Simkin PA, Nelp WB. Protein traffic in human synovial effusions. Arthritis Rheum 1987;30:57-63.

16 Jensen LT, Henriksen JH, Olesen HP, Risteli J, Lorenzen I. Lymphatic clearance of synovial fluid in conscious pigs; the aminoterminal propeptide of type III procollagen. Eur J Clin Invest 1993;23:778-84

17 Levick JR. An analysis of the interaction between extravascular plasma protein, interstitial flow and capillary filtration; application to synovium. Microvasc Res 1994;47:90-125.
18 Page-Thomas DP, Bard D, King B, Dingle JT. Clearance of proteoglycan from joint cavities. Ann Rheum Dis 1987;46: 934-7.

19 Levick JR, Price FM, Mason RM. Synovial matrix-synovial fluid system of joints. In: Comper WD, ed. Extracellular matrix. Vol 1. Tissue function. Amsterdam: Harwood Academic, 1996:328-77.

20 Patlak CS, Goldstein DA, Hoffman JF. The flow of solute and solvent across a two-membrane system. J Theor Biol 1963;5:426-42.

21 Levick JR, McDonald JN. Viscous and osmotically mediated changes of interstitial flow induced by extravascular albumin in synovium. Appendix. Microvasc Res 1994;47: $68-89$

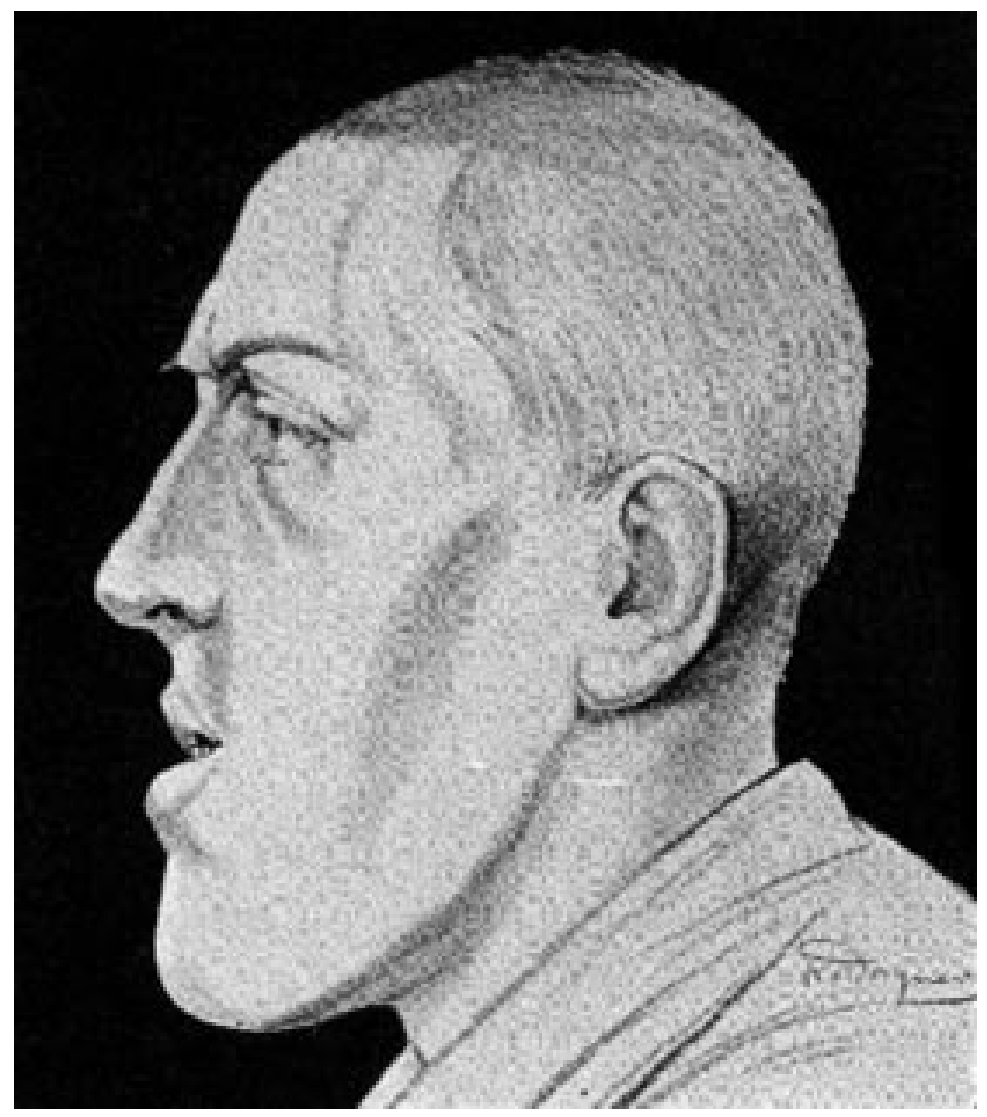

Figure 9 Acromegaly. Garnier M. Acromegaly. In: Marfan A-B, Apert, Aviragnet, Bernard L, Garnier M, Hallé F, Milian. Maladies des os. Paris: Librairie f-B Baillière et fils, 1912

Series editors $W$ Grassi, C Cervini 